\title{
Goal-oriented error estimation in the analysis of fluid flows with structural interactions
}

\author{
Thomas Grätsch, Klaus-Jürgen Bathe * \\ Department of Mechanical Engineering, Massachusetts Institute of Technology, 77 Massachusetts Avenue, Room 3-356, \\ Cambridge, MA 02139, United States
}

Received 15 March 2005; received in revised form 19 October 2005; accepted 20 October 2005

\begin{abstract}
We present some developments for the extension of goal-oriented error estimation procedures to the analysis of Navier-Stokes incompressible fluid flows with structural interactions. Particular focus is given on error assessment of specific quantities of interest defined on the structural part. The goal is to establish relatively coarse meshes to model the fluid flow but achieve acceptable accuracy in the quantities of interest. A nonlinear goal-oriented error estimation procedure is presented which is applicable to general nonlinear analyses. Some illustrative solutions using ADINA are given.
\end{abstract}

(C) 2005 Elsevier B.V. All rights reserved.

Keywords: Goal-oriented error estimation; Navier-Stokes fluid flow; Nonlinear structures; Interactions; FSI

\section{Introduction}

Since the pioneering developments of Argyris $[1,2],{ }^{1}$ and other researchers, the finite element method has been established as the primary analysis technique for structures, and has also been used to solve various multi-physics problems $[3,4]$. In particular, the analysis of fluid flows with structural interactions has been given increasing attention during the recent years. With the availability of numerical solution schemes for fluid flow response and structural response, a natural step is to pursue a fully coupled analysis. Such analyses are now performed in industry and academia to solve already quite complex problems.

However, as in all numerical solutions, the computational cost of solution and the accuracy reached for a given cost are important issues. These are particularly important considerations in the solution of Navier-Stokes fluid flows fully coupled to nonlinear structural response, like encountered, for example, in the analysis of motor car damping devices [5], in pumps and bearings [6], or fluid flows in biological systems [7], see also [3,4]. The cost of solution is, roughly, given by the number of nodes or grid points used for the fluid and structural discretizations. If a fully three-dimensional analysis is pursued, the number of grid points can be very large to represent the fluid flow and millions of equations are solved. Fully coupled fluid flow structural interaction analyses can then be computationally very expensive. However, in many such analyses, the objective is to predict only the structural response accurately, whereas the fluid flow is not needed with great accuracy.

The large number of equations in fluid flow structural interaction analyses are usually due to the representation of the fluid. Indeed, in order for interaction effects to be significant, the structure is frequently thin and can be represented as a

\footnotetext{
${ }^{*}$ Corresponding author. Tel.: +1 617253 6645; fax: +1 6172532275.

E-mail address: kjb@mit.edu (K.-J. Bathe).

${ }^{1}$ This paper was submitted in honor of J.H. Argyris.
} 
shell, hence a two-dimensional domain, not requiring, by far, as many grid points as the three-dimensional fluid domain. In essence then, it must be the aim to reduce the number of fluid elements or grid points in the discretization in order to reduce the solution cost. For this purpose, we have concentrated our research on the development of the flow-condition-based interpolation (FCBI) finite element solution approach for Navier-Stokes fluids [8-10]. The aim in this solution approach is to develop schemes for Navier-Stokes fluids at low and high Reynolds numbers that are stable and accurate, and reasonably accurate even when using rather coarse meshes. The accuracy should then increase optimally as the fluid mesh is refined.

The use of coarse meshes in the solution of fluid flows should be possible when only global quantities need be predicted accurately, like the overturning moment due to tractions/pressure on a structure. Of course, the level of accuracy must be sufficiently high to obtain the structural response to the desired accuracy, and to ensure that this is the case, the solution error needs to be measured and controlled. In principle, a number of error measures but in particular goaloriented error measures have the potential to be very useful [11-21]. Indeed, the basic aim with goal-oriented error measures, to assess the error in certain solution quantities, is directly applicable. The goal-oriented error measures might be applied to establish the error in the tractions on a structure, or more directly, to assess the error in the quantities of interest in the structure. Hence, our objective in fluid flow structural interaction analyses is to use the FCBI solution schemes in rather coarse meshes together with goal-oriented error measures to control the error of solution in the structural response.

Of course, to establish an effective coarse mesh of FCBI elements will in general require some iterations on the mesh. However, the expense of the iterations - if necessary - might be much less than simply running a very fine fluid mesh, in particular when the fluid mesh needs to be only established once for a structural optimization. The structural mesh could of course also be iterated upon, but in practice, the cost arising by use of a very fine (and therefore fixed) structural mesh is frequently relatively small.

The objective of this paper is to present some basic approaches to use goal-oriented error assessments in steady-state fluid flow structural interaction analyses in order to obtain acceptable accuracy in the structural response of interest with the use of coarse meshes to represent the fluid flow. While goal-oriented error estimation techniques have been presented for a large class of linear and nonlinear problems, see $[13,14,19,20,22]$ and the references therein, the actual application in practical analyses still needs to be explored [20], and the extension to fluid flow structural interaction problems, like considered in this paper, is still an open research task.

In the next sections of the paper, we first present the governing equations solved in the fully coupled Navier-Stokes fluid flow with the structure. We then focus on the nonlinear goal-oriented error estimation techniques developed to assess and control the error in the structural response of interest. Next we illustrate the use of the proposed procedures in some example solutions using ADINA [23,24] with the proposed error measure procedures implemented externally to this program. Finally, in the concluding remarks, we point out that the development given herein is surely only a start for much more research to be performed in this area, but the approach followed in this paper seems very promising.

\section{Governing equations of fully coupled fluid flow structure interaction}

In this section, we present the mathematical model and the finite element equations of the fluid flow structure interaction problem that we consider, see also Ref. [24].

\subsection{Fluid equations}

We consider an open bounded fluid domain $\Omega \in \mathbb{R}^{3}$ with boundary $\Gamma=\Gamma_{\mathrm{D}} \cup \Gamma_{\mathrm{N}}$, where $\Gamma_{\mathrm{D}}$ and $\Gamma_{\mathrm{N}}$ are the Dirichlet and Neumann boundaries, respectively, and $\Gamma_{i} \subset \Gamma_{\mathrm{D}}$ is the fluid-structure interface boundary. The incompressible Navier-Stokes equations of a steady-state fluid flow problem can be written in non-conservative form as

$$
\begin{aligned}
& -\nabla \cdot \tau+\rho(\mathbf{v} \cdot \nabla) \mathbf{v}=\mathbf{f} \quad \mathbf{x} \in \Omega, \\
& \nabla \cdot \mathbf{v}=0 \quad \mathbf{x} \in \Omega,
\end{aligned}
$$

subject to (sufficiently smooth) boundary conditions

$$
\begin{aligned}
& \mathbf{v}=\mathbf{0} \quad \mathbf{x} \in \Gamma_{i}, \\
& \mathbf{v}=\mathbf{v}_{\mathrm{D}} \quad \mathbf{x} \in \Gamma_{\mathrm{D}} \backslash \Gamma_{i}, \\
& \boldsymbol{\tau} \cdot \mathbf{n}=\mathbf{t} \quad \mathbf{x} \in \Gamma_{\mathrm{N}},
\end{aligned}
$$

where

$$
\tau=\tau(\mathbf{v}, p)=-p \mathbf{I}+\mu\left[\nabla \mathbf{v}+(\nabla \mathbf{v})^{\mathrm{T}}\right]
$$


is the stress tensor, $\mu$ is the viscosity, $\mathbf{v}$ are the velocities, $p$ is the pressure, $\mathbf{f}$ are body forces per unit volume, $\mathbf{v}_{\mathrm{D}}$ are the prescribed velocities on $\Gamma_{\mathrm{D}}, \mathbf{t}$ are the prescribed tractions on $\Gamma_{\mathrm{N}}, \mathbf{n}$ is the unit outward normal vector to the boundary surface of the fluid, and $\rho$ is the density. The variational formulation of the Navier-Stokes equations reads [25]:

Find $(\mathbf{v}, p) \in V \times P$ such that

$$
a((\mathbf{v}, p),(\mathbf{w}, q))=l(\mathbf{w}) \quad \forall(\mathbf{w}, q) \in V_{0} \times P,
$$

where

$$
\begin{aligned}
& a((\mathbf{v}, p),(\mathbf{w}, q))=\int_{\Omega} \tau \cdot \nabla \mathbf{w} \mathrm{d} \Omega+\int_{\Omega} \rho(\mathbf{v} \cdot \nabla) \mathbf{v} \cdot \mathbf{w} \mathrm{d} \Omega+\int_{\Omega} q \nabla \cdot \mathbf{v} \mathrm{d} \Omega, \\
& l(\mathbf{w})=\int_{\Omega} \mathbf{f} \cdot \mathbf{w} \mathrm{d} \Omega+\int_{\Gamma_{\mathrm{N}}} \mathbf{t} \cdot \mathbf{w} \mathrm{d} s
\end{aligned}
$$

and the Hilbert spaces are defined by

$$
\begin{aligned}
& V_{0}=\left\{\mathbf{w} \in\left[H^{1}(\Omega)\right]^{3}: \mathbf{w}=\mathbf{0} \text { on } \Gamma_{\mathrm{D}}\right\}, \\
& P=\left\{q \in L^{2}(\Omega)\right\} .
\end{aligned}
$$

The trial space $V$ differs from the test space $V_{0}$ in that functions of $V$ satisfy the inhomogeneous boundary data on $\Gamma_{\mathrm{D}}{ }^{2}$

Of course, the coupling between the fluid and the structure must satisfy the conditions of compatibility and traction equilibrium at the fluid-structure interface. In this work, the displacements of the structure are mapped onto the fluid domain, i.e.,

$$
\mathbf{u}=\mathbf{u}_{\mathrm{s}} \quad \mathbf{x} \in \Gamma_{i}
$$

and hence the fluid domain $\Omega=\Omega\left(\mathbf{u}_{\mathrm{s}}\right)$ is a function of the structural displacements $\mathbf{u}_{\mathrm{s}}$.

\subsection{Solid equations}

We consider an open bounded domain $\Omega_{\mathrm{s}} \in \mathbb{R}^{3}$ of a structure or solid with boundary $\Gamma_{\mathrm{s}}=\Gamma_{\mathrm{D}}^{\mathrm{s}} \cup \Gamma_{\mathrm{N}}^{\mathrm{s}}$ where $\Gamma_{\mathrm{D}}^{\mathrm{s}}$ and $\Gamma_{\mathrm{N}}^{\mathrm{s}}$ are the Dirichlet and Neumann boundaries, respectively, and $\Gamma_{i} \subset \Gamma_{\mathrm{N}}^{\mathrm{s}}$ is the fluid-structure interface boundary. The structural (or solid) response is described using a Lagrangian formulation where the structure can comprise any structural elements such as beams, plates, or shells. The structural deformations can be complex and include large deformations and rotations. Considering a general 3D-nonlinear response, the governing equilibrium equations are

$$
\nabla \cdot \tau_{\mathrm{s}}+\mathbf{f}_{\mathrm{s}}=\mathbf{0} \quad \mathbf{x} \in \Omega_{\mathrm{s}}
$$

associated with the boundary conditions

$$
\begin{aligned}
& \mathbf{u}_{\mathrm{s}}=\mathbf{0} \quad \mathbf{x} \in \Gamma_{\mathrm{D}}^{\mathrm{s}}, \\
& \tau_{\mathrm{s}} \cdot \mathbf{n}_{\mathrm{s}}=\mathbf{t}_{\mathrm{s}} \quad \mathbf{x} \in \Gamma_{\mathrm{N}}^{\mathrm{s}} \backslash \Gamma_{i}, \\
& \tau_{\mathrm{s}} \cdot \mathbf{n}_{\mathrm{s}}=-\tau \cdot \mathbf{n} \quad \mathbf{x} \in \Gamma_{i},
\end{aligned}
$$

where $\tau_{\mathrm{s}}$ is the structural stress tensor, $\mathbf{f}_{\mathrm{s}}$ are body forces per unit volume, $\mathbf{t}_{\mathrm{s}}$ are tractions applied on $\Gamma_{\mathrm{N}}^{\mathrm{s}}$, and $\mathbf{n}_{\mathrm{s}}$ is the unit outward normal vector to the boundary surface of the structure. The stresses are of course evaluated using the relevant constitutive relation (we considered in this research an elastic behavior). We note that in Eq. (16) the traction equilibrium between the fluid and the structure is satisfied on $\Gamma_{i}$, that is, the tractions of the fluid are mapped onto the structure.

The variational formulation of this problem can be written as [25]:

Find $\mathbf{u}_{\mathrm{s}} \in V_{\mathrm{s}}$ such that

$$
a_{\mathrm{s}}\left(\mathbf{u}_{\mathrm{s}}, \mathbf{v}_{\mathrm{s}}\right)=l_{\mathrm{s}}\left(\mathbf{v}_{\mathrm{s}}\right) \quad \forall \mathbf{v}_{\mathrm{s}} \in V_{\mathrm{s}},
$$

where

$$
\begin{aligned}
& a_{\mathrm{s}}\left(\mathbf{u}_{\mathrm{s}}, \mathbf{v}_{\mathrm{s}}\right)=\int_{\Omega_{\mathrm{s}}} \tau_{\mathrm{s}} \cdot \boldsymbol{\epsilon}_{\mathrm{s}} \mathrm{d} \Omega \\
& l_{\mathrm{s}}\left(\mathbf{v}_{\mathrm{s}}\right)=\int_{\Omega_{\mathrm{s}}} \mathbf{f}_{\mathrm{s}} \cdot \mathbf{v}_{\mathrm{s}} \mathrm{d} \Omega+\int_{\Gamma_{\mathrm{N}}^{\mathrm{s}} \backslash \Gamma_{i}} \mathbf{t}_{\mathrm{s}} \cdot \mathbf{v}_{\mathrm{s}} \mathrm{d} s-\int_{\Gamma_{i}}(\tau \cdot \mathbf{n}) \cdot \mathbf{v}_{\mathrm{s}} \mathrm{d} s
\end{aligned}
$$

with strain tensor $\epsilon_{\mathrm{s}}$ and the Hilbert space is defined by

$$
V_{\mathrm{s}}=\left\{\mathbf{v}_{\mathrm{s}} \in\left[H^{1}\left(\Omega_{\mathrm{s}}\right)\right]^{3}: \mathbf{v}_{\mathrm{s}}=\mathbf{0} \text { on } \Gamma_{\mathrm{D}}^{\mathrm{s}}\right\} .
$$

\footnotetext{
${ }^{2}$ Actually, to be precise, $V$ is not a linear space, but an affine manifold that can be thought of as obtained by translating the linear space $V_{0}$.
} 


\subsection{Coupling between fluid and structure}

The coupling between the fluid and the structure is based on an arbitrary-Lagrangian-Eulerian formulation for the fluid that is coupled to the Lagrangian formulation of the structure [24].

Using the variational formulations for the fluid flow problem and the structural part problem, the nonlinear coupled problem can be written in compact form as:

Find $\left\{\mathbf{v}, p, \mathbf{u}_{\mathrm{s}}\right\} \in \mathscr{V}=V \times P \times V_{\mathrm{s}}$ such that

$$
a((\mathbf{v}, p),(\mathbf{w}, q))+a_{\mathrm{s}}\left(\mathbf{u}_{\mathrm{s}}, \mathbf{v}_{\mathrm{s}}\right)=l(\mathbf{w})+l_{\mathrm{s}}\left(\mathbf{v}_{\mathrm{s}}\right) \quad \forall\left\{\mathbf{w}, q, \mathbf{v}_{\mathrm{s}}\right\} \in \mathscr{V}_{0},
$$

where $\mathscr{V}_{0}=V_{0} \times P \times V_{\mathrm{s}}$. This nonlinear variational problem (21) describes a fully coupled fluid flow interaction problem. The fluid domain, on which $a((.,),.(.,)$.$) is defined, depends on the structural displacements \mathbf{u}_{\mathrm{s}}$, while the tractions of the fluid are mapped onto the structure.

\section{Nonlinear goal-oriented error estimation}

In this section, we propose a framework for general nonlinear goal-oriented error estimation which can be used with existing finite element programs and which is applicable to a wide range of applications including fluid flows interacting with general nonlinear structural behavior.

\subsection{General framework}

We develop our approach for general nonlinear goal-oriented error estimation. For simplicity we consider first the case of homogeneous boundary conditions on the Dirichlet boundary and address the case of non-homogeneous boundary data in Section 3.3. We begin with the abstract nonlinear variational problem [25]:

Find $\mathbf{U} \in \mathscr{V}_{0}$ such that

$$
A(\mathbf{U} ; \mathbf{V})=L(\mathbf{V}) \quad \forall \mathbf{V} \in \mathscr{V}_{0},
$$

where $\mathbf{U}$ is the exact (weak) solution, $A(. ;$.) is a semi-linear form that is linear only in the second argument, $L($.) is a linear functional, and $\mathscr{V}_{0}$ is the appropriate Hilbert space. For instance, in case of fluid flow structure interaction, these forms are given by

$$
\begin{aligned}
& A(\mathbf{U} ; \mathbf{V})=a((\mathbf{v}, p),(\mathbf{w}, q))+a_{\mathrm{s}}\left(\mathbf{u}_{\mathrm{s}}, \mathbf{v}_{\mathrm{s}}\right), \\
& L(\mathbf{V})=l(\mathbf{w})+l_{\mathrm{s}}\left(\mathbf{v}_{\mathrm{s}}\right) .
\end{aligned}
$$

The corresponding finite element approximation is based on finite dimensional subspaces $\mathscr{V}_{0, h} \subset \mathscr{V}_{0}$ to determine $\mathbf{U}_{h} \in \mathscr{V}_{0, h}$ such that

$$
A\left(\mathbf{U}_{h} ; \mathbf{V}_{h}\right)=L\left(\mathbf{V}_{h}\right) \quad \forall \mathbf{V}_{h} \in \mathscr{V}_{0, h} .
$$

To solve the equations we can employ the "directly fully coupled solution" or the "iterative solution by partitioning", and the same solution is obtained in either case [24,25].

A measure of the solution error $\mathbf{E}_{h}=\mathbf{U}-\mathbf{U}_{h}$ is given by the residual [20]

$$
\mathscr{R}\left(\mathbf{U}_{h} ; \mathbf{V}\right)=L(\mathbf{V})-A\left(\mathbf{U}_{h} ; \mathbf{V}\right) \quad \forall \mathbf{V} \in \mathscr{V}_{0},
$$

with the Galerkin orthogonality

$$
\mathscr{R}\left(\mathbf{U}_{h} ; \mathbf{V}_{h}\right)=L\left(\mathbf{V}_{h}\right)-A\left(\mathbf{U}_{h} ; \mathbf{V}_{h}\right)=0 \quad \forall \mathbf{V}_{h} \in \mathscr{V}_{0, h} .
$$

Our goal is to calculate a specific quantity of the solution space, denoted by $Q(\mathbf{U})$, which is a linear or nonlinear functional defined in the dual space $\mathscr{V}_{0}^{\prime}$. This so-called quantity of interest can be almost any linear or nonlinear local or global quantity of the solution, such as the square of the $L^{2}$-norm of the solution

$$
Q(\mathbf{U})=\int_{\Omega_{\mathrm{c}}} \mathbf{U} \cdot \mathbf{U d} \Omega
$$

(with $\Omega_{\mathrm{c}}$ denoting the domain of the coupled problem) or, in the case of fluid flow structure interaction, the tractions corresponding to the fluid flow acting on the fluid-structure interface

$$
Q(\mathbf{U})=\int_{\Gamma_{i}} \tau(\mathbf{v}, p) \mathbf{n} \mathrm{d} s .
$$


For the purpose of goal-oriented error estimation we (frequently) assume that the following Gateaux-derivatives of the semi-linear form and the quantity of interest exist

$$
\begin{aligned}
& A^{\prime}(\mathbf{U} ; \boldsymbol{\varphi}, \mathbf{V})=\left[\frac{\mathrm{d}}{\mathrm{d} \varepsilon} A(\mathbf{U}+\varepsilon \boldsymbol{\varphi} ; \mathbf{V})\right]_{\varepsilon=0}, \\
& Q^{\prime}(\mathbf{U} ; \mathbf{V})=\left[\frac{\mathrm{d}}{\mathrm{d} \varepsilon} Q(\mathbf{U}+\varepsilon \mathbf{V})\right]_{\varepsilon=0} .
\end{aligned}
$$

Clearly, $A^{\prime}(. ; .,$.$) is the tangent form used in the nonlinear iteration to solve (22) which is a bilinear form in the last two$ arguments, but not necessarily symmetric in $\boldsymbol{\varphi}$ and $\mathbf{V}$. The key for estimating the error in a quantity of interest is for us to consider for a specified $\mathbf{U}$ a (linear) auxiliary problem:

Find $\mathbf{Z} \in \mathscr{V}_{0}$ such that

$$
A^{\prime}(\mathbf{U} ; \mathbf{Z}, \mathbf{V})=Q^{\prime}(\mathbf{U} ; \mathbf{V}) \quad \forall \mathbf{V} \in \mathscr{V}_{0},
$$

in which $\mathbf{Z}$ denotes the influence function corresponding to the choice of the quantity of interest obtained at the "linearization point" of $\mathbf{U}$. However, since the exact solution $\mathbf{U}$ is generally unknown, the auxiliary problem is linearized around the finite element solution $\mathbf{U}_{h}$, which leads to the variational problem:

Find $\mathbf{Z} \in \mathscr{V}_{0}$ such that ${ }^{3}$

$$
A^{\prime}\left(\mathbf{U}_{h} ; \mathbf{Z}, \mathbf{V}\right)=Q^{\prime}\left(\mathbf{U}_{h} ; \mathbf{V}\right) \quad \forall \mathbf{V} \in \mathscr{V}_{0} .
$$

Then we may seek the error in the quantity of interest by choosing $\mathbf{V}=\mathbf{W}-\mathbf{U}_{h}$ which can give the starting point of a subsequent goal-oriented error estimate:

$$
A^{\prime}\left(\mathbf{U}_{h} ; \mathbf{Z}, \mathbf{W}-\mathbf{U}_{h}\right)=Q^{\prime}\left(\mathbf{U}_{h} ; \mathbf{W}-\mathbf{U}_{h}\right) .
$$

The quantity $Q^{\prime}\left(\mathbf{U}_{h} ; \mathbf{W}-\mathbf{U}_{h}\right)$ is a linear approximation of the true error in the quantity of interest evaluated at the linearization point, where $\mathbf{W}$ denotes a linear approximation of the exact solution $\mathbf{U}$ at this point.

Remark 1. In the case of a symmetric tangent form, the following dual problem can be considered:

Find $\mathbf{Z} \in \mathscr{V}_{0}$ such that

$$
A^{\prime}(\mathbf{U} ; \mathbf{V}, \mathbf{Z})=Q^{\prime}(\mathbf{U} ; \mathbf{V}) \quad \forall \mathbf{V} \in \mathscr{V}_{0}
$$

Of course, this problem is in this case identical to the auxiliary problem we consider in (32) and the same solutions are obtained.

Remark 2. In case of a symmetric tangent form we also have the relation

$$
Q^{\prime}\left(\mathbf{U}_{h} ; \mathbf{U}_{h}\right)=A^{\prime}\left(\mathbf{U}_{h} ; \mathbf{Z}_{h}, \mathbf{U}_{h}\right)=A^{\prime}\left(\mathbf{U}_{h} ; \mathbf{U}_{h}, \mathbf{Z}_{h}\right)=L\left(\mathbf{Z}_{h}\right),
$$

where $Q^{\prime}\left(\mathbf{U}_{h} ; \mathbf{U}_{h}\right)$ is a Gateaux linearization of $Q\left(\mathbf{U}_{h}\right)$ at the linearization point and we use the corresponding influence function in the evaluation of $L\left(\mathbf{Z}_{h}\right)$.

Remark 3. Since $Q^{\prime}\left(\mathbf{U}_{h} ; \mathbf{U}_{h}\right)$ is actually used in the assessment of the error, see (34), it is interesting to evaluate the difference

$$
d=\left|Q\left(\mathbf{U}_{h}\right)-Q^{\prime}\left(\mathbf{U}_{h} ; \mathbf{U}_{h}\right)\right|
$$

as a measure for degree of nonlinearity of the problem with respect to the quantity of interest. For instance, considering the quantity of interest defined in (28), we obtain

$$
\begin{aligned}
& Q\left(\mathbf{U}_{h}\right)=\int_{\Omega_{\mathrm{c}}} \mathbf{U}_{h}^{2} \mathrm{~d} \Omega, \\
& Q^{\prime}\left(\mathbf{U}_{h} ; \mathbf{U}_{h}\right)=\int_{\Omega_{\mathrm{c}}} 2 \mathbf{U}_{h}^{2} \mathrm{~d} \Omega,
\end{aligned}
$$

so that a subsequent error estimate based on $Q^{\prime}\left(\mathbf{U}_{h} ; \mathbf{W}-\mathbf{U}_{h}\right)$ neglects higher-order terms in the error $Q(\mathbf{W})-Q\left(\mathbf{U}_{h}\right)$, even if $\mathbf{W}$ were equal to $\mathbf{U}$. Of course, in case of a linear quantity of interest, such as the one in (29), we have $d=0$.

\footnotetext{
${ }^{3}$ Note that the solutions of (32) and (33) are of course different, but it will be apparent in subsequent equations which $\mathbf{Z}$ is meant.
} 


\subsection{A strategy for computing the influence function}

We are largely interested in a procedure that can be used externally-coupled to existing finite element programs, and therefore suggest to replace the Gateaux-derivative of the semi-linear form given on the left-hand side in (33) by

$$
\begin{aligned}
Q^{\prime}\left(\mathbf{U}_{h} ; \mathbf{V}\right) & =A^{\prime}\left(\mathbf{U}_{h} ; \mathbf{Z}, \mathbf{V}\right) \\
& =\left.\frac{\mathrm{d}}{\mathrm{d} \varepsilon} A\left(\mathbf{U}_{h}+\varepsilon \mathbf{Z} ; \mathbf{V}\right)\right|_{\varepsilon=0} \\
& \cong \varepsilon^{-1}\left[A\left(\mathbf{U}_{h}+\varepsilon \mathbf{Z} ; \mathbf{V}\right)-A\left(\mathbf{U}_{h} ; \mathbf{V}\right)\right] .
\end{aligned}
$$

In order to obtain a finite element solution of the influence function, we choose $\varepsilon$ to be a "scaling factor" of the linear auxiliary problem and solve the variational problem:

Find $\mathbf{U}_{h}+\varepsilon \mathbf{Z}_{h} \in \mathscr{V}_{0, h}$ such that

$$
A\left(\mathbf{U}_{h}+\varepsilon \mathbf{Z}_{h} ; \mathbf{V}_{h}\right)=L\left(\mathbf{V}_{h}\right)+\varepsilon Q^{\prime}\left(\mathbf{U}_{h} ; \mathbf{V}_{h}\right) \quad \forall \mathbf{V}_{h} \in \mathscr{V}_{0, h} .
$$

After establishing the solution of (43) the influence function is calculated by

$$
\mathbf{Z}_{h}=\varepsilon^{-1}\left[\left(\mathbf{U}_{h}+\varepsilon \mathbf{Z}_{h}\right)-\mathbf{U}_{h}\right]
$$

and the so-constructed tangent form is denoted by

$$
A_{\varepsilon}^{\prime}\left(\mathbf{U}_{h} ; \mathbf{Z}_{h}, \mathbf{V}\right)=\varepsilon^{-1}\left[A\left(\mathbf{U}_{h}+\varepsilon \mathbf{Z}_{h} ; \mathbf{V}\right)-A\left(\mathbf{U}_{h} ; \mathbf{V}\right)\right] .
$$

Remark 4. To solve (43) we first apply the load $L\left(\mathbf{V}_{h}\right)$ of the primal problem in an appropriate number of steps. After obtaining the solution $\mathbf{U}_{h}$ we apply the load term $\varepsilon Q^{\prime}\left(\mathbf{U}_{h} ; \mathbf{V}_{h}\right)$ in one additional step. The parameter $\varepsilon$ is a very small number and can be adaptively chosen such that there is only a linear contribution of $\varepsilon \mathbf{Z}_{h}$ to the solution. The computational cost of the proposed technique is small since there is only one step needed for applying $\varepsilon Q^{\prime}\left(\mathbf{U}_{h} ; \mathbf{V}_{h}\right)$. Hence, for the evaluation of the difference in (44) we simply take the solutions $\left(\mathbf{U}_{h}+\varepsilon \mathbf{Z}_{h}\right)$ and $\mathbf{U}_{h}$ obtained in the same finite element analysis of the problem.

\subsection{Error control for problems with symmetric tangent forms}

Many nonlinear variational problems in solid mechanics and some problems in fluid mechanics result in a symmetric tangent form. In these cases, or for any other problems with an underlying symmetrical tangent form, we proceed for the purpose of goal-oriented error estimation as follows:

$$
\begin{aligned}
Q^{\prime}\left(\mathbf{U}_{h} ; \mathbf{W}-\mathbf{U}_{h}\right) & =Q^{\prime}\left(\mathbf{U}_{h} ; \mathbf{W}\right)-Q^{\prime}\left(\mathbf{U}_{h} ; \mathbf{U}_{h}\right) \\
& =A^{\prime}\left(\mathbf{U}_{h} ; \mathbf{Z}, \mathbf{W}\right)-A^{\prime}\left(\mathbf{U}_{h} ; \mathbf{Z}_{h}, \mathbf{U}_{h}\right) \\
& \approx L(\mathbf{Z})-A_{\varepsilon}^{\prime}\left(\mathbf{U}_{h} ; \mathbf{Z}_{h}, \mathbf{U}_{h}\right),
\end{aligned}
$$

where the approximate sign in (48) refers to replacing the Gateaux-derivative of the second form in (47) by the finite difference expression using (45). We note that since $L($.) is per definition a linear functional, we use in the step from (47) to (48)

$$
A^{\prime}\left(\mathbf{U}_{h} ; \mathbf{Z}, \mathbf{W}\right)=L(\mathbf{Z})
$$

with $A^{\prime}(. ; .,$.$) as defined in (30).$

To actually obtain a computable quantity of the error in the quantity of interest, one useful strategy (and commonly accepted practice) is to replace the unknown solution $\mathbf{Z}$ by a solution obtained using a richer space than $\mathscr{V}_{0, h}$ : For instance, Grätsch and Bathe $[20,21]$ used the same mesh of 4-node and 9-node elements in shell analyses. Hence, if in a 2D or shell analysis $\mathbf{Z}_{h}^{9 \text {-node }}$ denotes the 9-node element solution and $\mathbf{Z}_{h}$ the 4-node element solution ( $h$ denotes the element size in each case), we obtain from (48)

$$
Q^{\prime}\left(\mathbf{U}_{h} ; \mathbf{W}-\mathbf{U}_{h}\right) \approx L\left(\mathbf{Z}_{h}^{9-\text { node }}\right)-A_{\varepsilon}^{\prime}\left(\mathbf{U}_{h} ; \mathbf{Z}_{h}, \mathbf{U}_{h}\right)
$$

Here the solutions $\mathbf{Z}_{h}$ and $\mathbf{Z}_{h}^{9-\text { node }}$ are computed using (43) and (44) with the mesh of 4-node and 9-node elements, respectively.

For the purpose of driving mesh adaptivity, we then obtain local mesh refinement indicators by considering

$$
Q^{\prime}\left(\mathbf{U}_{h} ; \mathbf{W}-\mathbf{U}_{h}\right) \approx \sum_{K}\left\{L\left(\mathbf{Z}_{h}^{9 \text {-node }}\right)_{K}-A_{\varepsilon}^{\prime}\left(\mathbf{U}_{h} ; \mathbf{Z}_{h}, \mathbf{U}_{h}\right)_{K}\right\}
$$


where $K$ denotes the volume of an element in the finite element mesh domain. Note that the estimates in (50) and (51) are very similar to those obtained in the standard duality approach $[19,20]$, where the Gateaux-derivative $A^{\prime}(. ; .,$.$) is employed$ instead of $A_{\varepsilon}^{\prime}(. ; .,$.$) used here and further integration by parts is applied to recast the estimate in terms of element residuals$ and jump terms across interelement boundaries.

For calculating the error for a given mesh only, we may use the simple relation

$$
Q^{\prime}\left(\mathbf{U}_{h} ; \mathbf{W}-\mathbf{U}_{h}\right) \approx L\left(\mathbf{Z}_{h}^{9 \text {-node }}\right)-L\left(\mathbf{Z}_{h}\right)
$$

which for a linear problem reads

$$
Q\left(\mathbf{U}-\mathbf{U}_{h}\right) \approx L\left(\mathbf{Z}_{h}^{9 \text {-node }}\right)-L\left(\mathbf{Z}_{h}\right) .
$$

We once again emphasize that to obtain the higher-order approximation $\mathbf{Z}_{h}^{9-\text {-node }}$, the same mesh is used as in the calculation of $\mathbf{Z}_{h}[20,21]$.

So far we (implicitly) assumed that there are no inhomogeneous data applied at the Dirichlet part of the boundary. In case $\mathbf{U} \neq \mathbf{0}$ on $\Gamma_{\mathrm{D}}$ we actually consider the variational problem:

Find $\mathbf{U} \in \mathscr{V}=V \times P \times V_{\mathrm{s}}$ such that

$$
A(\mathbf{U} ; \mathbf{V})=L(\mathbf{V}) \quad \forall \mathbf{V} \in \mathscr{V}_{0},
$$

where the trial space $\mathscr{V}$ differs from the test space $\mathscr{V}_{0}$ in that functions of $\mathscr{V}$ satisfy the inhomogeneous boundary data on $\Gamma_{\mathrm{D}}$.

For obtaining an error estimate similar to (52) we split the solution $\mathbf{U}$ into two parts

$$
\mathbf{U}=\tilde{\mathbf{U}}+\mathbf{U}^{\Gamma},
$$

where $\tilde{\mathbf{U}}$ denotes the solution in $\mathscr{V}_{0}$ for homogeneous boundary data and $\mathbf{U}^{\Gamma}$ is chosen such that $\mathbf{U}^{T}=\mathbf{U}_{\mathrm{D}}$ on $\Gamma_{\mathrm{D}}$ with $\mathbf{U}_{\mathrm{D}}$ denoting the inhomogeneous boundary data on $\Gamma_{\mathrm{D}}$ (a reasonable choice of $\mathbf{U}^{T}$ ensures the effectiveness of the solution) [25]. Employing (55) in (54) leads to

$$
A\left(\tilde{\mathbf{U}}+\mathbf{U}^{\Gamma} ; \mathbf{V}\right)=L(\mathbf{V}) .
$$

The solution $\tilde{\mathbf{U}}$ then satisfies the variational problem:

Find $\tilde{\mathbf{U}} \in \mathscr{V}_{0}=V_{0} \times P \times V_{\mathrm{s}}$ such that

$$
A^{\star}(\tilde{\mathbf{U}} ; \mathbf{V})=L^{\star}(\mathbf{V}) \quad \forall \mathbf{V} \in \mathscr{V}_{0}
$$

where the modified forms $A^{\star}(. ;$.$) and L^{\star}($.) account for the inhomogeneous data at the Dirichlet boundary. The error estimation can now directly proceed as described above.

Remark 5. However, it is important that in the solution of the auxiliary problem defined in (32), or its finite element approximation, we now apply zero boundary conditions on that portion of the boundary $\Gamma_{\mathrm{D}}$ on which inhomogeneous boundary data are prescribed in the primal problem. For example, when applying an inlet velocity $\mathbf{v}_{\mathrm{D}}$ on a portion $\Gamma_{\text {in }} \subset \Gamma_{\mathrm{D}}$ of the Dirichlet boundary, for solving the auxiliary problem we have to apply the boundary condition $\mathbf{v}=\mathbf{0}$ on $\Gamma_{\text {in }}$, that is, the inlet has been closed (see also Section 4.1).

\subsection{Error control for problems with non-symmetric tangent forms}

In case of a non-symmetric tangent form $A^{\prime}(. ; .,$.$) the step from (47) to (48) is not possible, hence the error representa-$ tions (50)-(52) can not be used. One possible way to proceed is to start with the residual (26) and choosing $\mathbf{V}=\mathbf{Z}-\mathbf{Z}_{h}$ yields

$$
\mathscr{R}\left(\mathbf{U}_{h} ; \mathbf{Z}-\mathbf{Z}_{h}\right)=L\left(\mathbf{Z}-\mathbf{Z}_{h}\right)-A\left(\mathbf{U}_{h} ; \mathbf{Z}-\mathbf{Z}_{h}\right) .
$$

Then we can also define

$$
\overline{\mathscr{R}}\left(\mathbf{U}_{h}, \mathbf{Z}_{h} ; \mathbf{V}\right)=Q^{\prime}\left(\mathbf{U}_{h} ; \mathbf{V}\right)-A^{\prime}\left(\mathbf{U}_{h} ; \mathbf{Z}_{h}, \mathbf{V}\right) \quad \forall \mathbf{V} \in \mathscr{V}_{0} .
$$

These relations are used in $[19,22]$ to establish an error measure. However, we can also use the exact error representation

$$
Q\left(\mathbf{E}_{h}\right)=Q(\mathbf{U})-Q\left(\mathbf{U}_{h}\right)
$$

and when a linear quantity of interest and local error indicators are sought

$$
Q\left(\mathbf{E}_{h}\right)=A(\mathbf{Z} ; \mathbf{U})-A\left(\mathbf{Z}_{h} ; \mathbf{U}_{h}\right),
$$


where $\mathbf{Z}$ and $\mathbf{Z}_{h}$ are solutions of nonlinear variational problems and are not influence functions of $Q(\mathbf{U})$ and $Q\left(\mathbf{U}_{h}\right)$ in the meaning of linear analysis.

Using this approach, a useful strategy is to replace the unknown solution $\mathbf{Z}$ by a higher-order approximation. For example, as considered already above, in two-dimensional analyses of fluid flow structure interactions, we may use for the Navier-Stokes fluid 4-node elements to calculate $\mathbf{Z}_{h}$ and then use the same mesh of 9-node elements to calculate $\mathbf{Z}_{h}^{9 \text {-node }}$, to obtain the approximate error representation

$$
Q(\mathbf{U})-Q\left(\mathbf{U}_{h}\right) \approx A\left(\mathbf{Z}_{h}^{9 \text {-node }} ; \mathbf{U}_{h}^{9 \text {-node }}\right)-A\left(\mathbf{Z}_{h} ; \mathbf{U}_{h}\right) .
$$

For the purpose of driving mesh adaptivity, we then obtain local mesh refinement indicators by considering

$$
Q(\mathbf{U})-Q\left(\mathbf{U}_{h}\right) \approx \sum_{K}\left\{A\left(\mathbf{Z}_{h}^{9 \text {-node }} ; \mathbf{U}_{h}^{9 \text {-node }}\right)_{K}-A\left(\mathbf{Z}_{h} ; \mathbf{U}_{h}\right)_{K}\right\} .
$$

The error representation (61) and its approximation (62) are quite general but an even more general approximate error estimate, that includes the case above but in particular is directly applicable to nonlinear goal quantities, is given by

$$
Q(\mathbf{U})-Q\left(\mathbf{U}_{h}\right) \approx Q\left(\mathbf{U}_{h}^{9 \text {-node }}\right)-Q\left(\mathbf{U}_{h}\right),
$$

where the quantity of interest is directly evaluated using $\mathbf{U}_{h}^{9-\text { node }}$ and $\mathbf{U}_{h}$.

Interpreting $\mathbf{U}_{h}^{9-\text {-node }}$ and $\mathbf{Z}_{h}^{9 \text {-node }}$ in general as higher-order element solutions obtained with the same mesh as used to calculate $\mathbf{U}_{h}$ and $\mathbf{Z}_{h}$, the error estimates in (62)-(64) are applicable to arbitrary nonlinear fully-coupled multi-physics problems while considering almost any quantity of interest.

\section{Numerical experiments}

In our numerical experiments, we employ the goal-oriented error estimation procedures for linear Stokes flow $(R e=0)$ with a rigid structure, to illustrate the concept of using influence functions, and Navier-Stokes fluid flow $(R e=200)$ interacting with a general nonlinear elastic structure. In both illustrative examples we focus on the tractions acting on the structural part of the problem, and we only estimate the error for uniform meshes and do not employ adaptivity. The original flow problems are solved using the 4-node FCBI elements available in ADINA. The error estimate then uses the fluid flow approximation obtained with a 9-node Galerkin finite element (using the 9 nodes for the velocities and the 4 corner nodes for the pressure interpolations) ${ }^{4}$ and the same mesh (i.e., with the same number of elements) as used with the 4-node FCBI elements. ${ }^{5}$ Hence, we simply change the element formulation in ADINA. For measuring the accuracy of our error estimate we use the effectivity index, which is the ratio between the estimated error and a reference error obtained using a very fine reference mesh.

\subsection{Flow over backward facing step and impinging on a rigid cantilever}

Our model problem is the fluid flow problem shown in Fig. 1(a) which has also been studied in [24]. We assume the cantilever to be rigid and consider linear Stokes flow. The quantities of interest are the horizontal tractions across the rigid cantilever located at $y=16.0$ and the resulting bending moment at the support

$$
\begin{aligned}
& Q_{1}(\mathbf{U})=\left.\int_{0}^{2}(\tau(\mathbf{v}, p) \mathbf{n})_{y} \mathrm{~d} z\right|_{y=16}, \\
& Q_{2}(\mathbf{U})=\left.\int_{0}^{2} z(\tau(\mathbf{v}, p) \mathbf{n})_{y} \mathrm{~d} z\right|_{y=16} .
\end{aligned}
$$

The finite element start-mesh consists of 488 two-dimensional 4-node FCBI elements (see Fig. 1(b)) and we choose $v_{0}=1$. For this mesh we want to study the influence function for the quantity of interest defined in (65).

An important point is to choose proper boundary conditions for the influence function problem. In the case of prescribed tractions at the channel inlet in the primal problem, we have to use zero traction boundary conditions in the influence function problem. However, in the case of prescribed velocities at the channel inlet (as used in this example), we have to apply zero-velocity boundary conditions at the inlet in the calculation of the influence function (see (57)).

\footnotetext{
${ }^{4}$ Also referred to as the Taylor-Hood, $Q 9 / Q 4$, or 9/4-c element [25].

${ }^{5}$ Ideally, we would be able to use here a 9-node FCBI element with much better stability and (almost) the same accuracy properties as provided by the Galerkin element, with the aim to also have the error control with coarse meshes for higher Re number flows. We are working on such an element [26], see also $[27,28]$.
} 


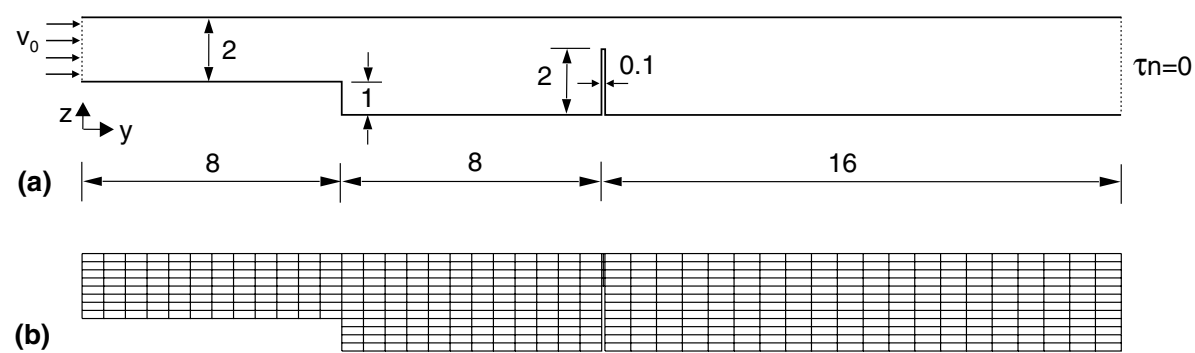

Fig. 1. Fluid flow structure interaction problem: (a) definition of the problem, cantilever is rigid in linear Stokes fluid flow and flexible in Navier-Stokes fluid flow, (b) start-mesh of four-node FCBI elements for the case of a rigid cantilever.

Fig. 2(a) and (b) show the velocities and the pressures of the influence function in case of inlet tractions in the primal problem, and Fig. 2(c) and (d) show the velocities and the pressures of the influence function in case of inlet velocities in the primal problem.

As expected, the numerical solution shows that employing the linear relationship

$$
Q\left(\tilde{\mathbf{U}}_{h}\right)=A^{\star}\left(\mathbf{Z}_{h}, \tilde{\mathbf{U}}_{h}\right)=A^{\star}\left(\tilde{\mathbf{U}}_{h}, \mathbf{Z}_{h}\right)=L^{\star}\left(\mathbf{Z}_{h}\right)=\int_{\Gamma_{\mathrm{D}}} \tau_{z}^{h} \mathbf{n} \cdot \mathbf{v}_{0} \mathrm{~d} s
$$

yields the same quantity of interest as obtained by calculating the finite element solution of the primal problem as usual and simply evaluating the quantity of interest on the solution space. In $(67) A^{\star}(.,$.$) denotes the symmetric bilinear$ form corresponding to the variational formulation of linear Stokes flow, $\mathbf{v}_{0}$ is the inlet velocity of the primal problem, and $L^{\star}($.) is the modified load term that accounts for the inhomogeneous data at the Dirichlet boundary (see Section $3.3)$.

We next want to estimate the error in the quantities of interest given in (65) and (66) employing the goal-oriented error estimation technique (53). The reference solution for assessing the error was obtained using a uniform mesh of 31,232 9-node Galerkin finite elements with 280,334 degrees of freedom. Using the start-mesh shown in Fig. 1(b) and employing the 4-node FCBI element in the analyses, the meshes are uniformly refined. As seen in Fig. 3, for both quantities, the estimated relative percentage errors decrease reasonably and the corresponding effectivity indices are close to 1.0 .

It is remarkable that even for a very coarse mesh (obtained by uniformly coarsening the start-mesh) of only 122 4-node FCBI elements (357 degrees of freedom) the solution is still useful yielding a relative error of $21.4 \%$ and $18.1 \%$ in the quantities of interest with corresponding effectivity indices of 1.42 and 1.77 for $Q_{1}(\mathbf{U})$ and $Q_{2}(\mathbf{U})$, respectively.
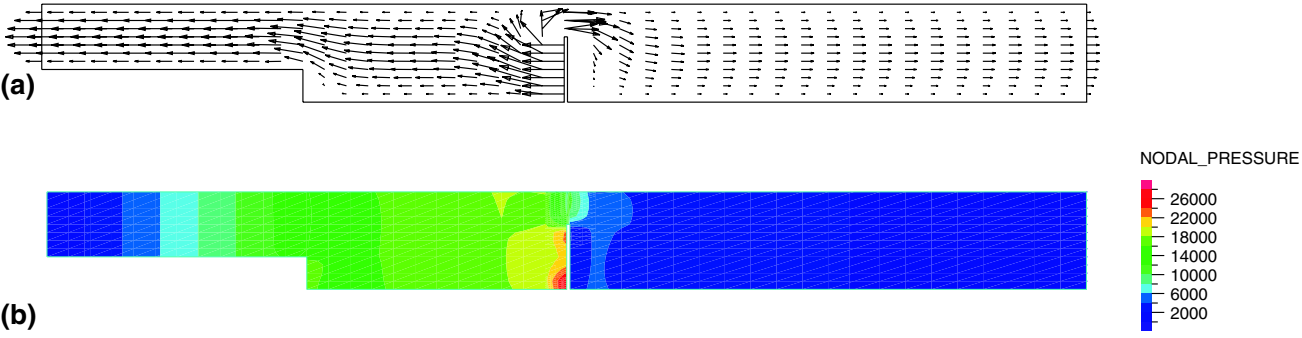

(c)
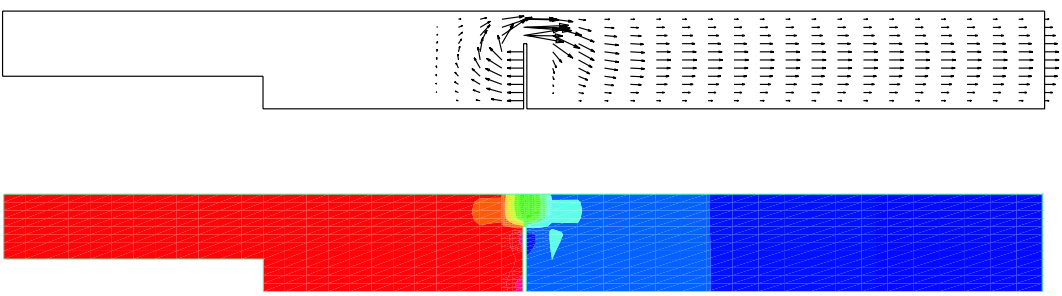

NODAL_PRESSURE

(d)

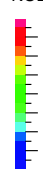

Fig. 2. Influence functions for the quantity of interest defined in (65) for the flow over backward facing step problem assuming a rigid cantilever: Primal problem consists of tractions at channel inlet, (a) velocities, (b) corresponding pressure field; primal problem consists of velocities at channel inlet, (c) velocities, (d) corresponding pressure field. 

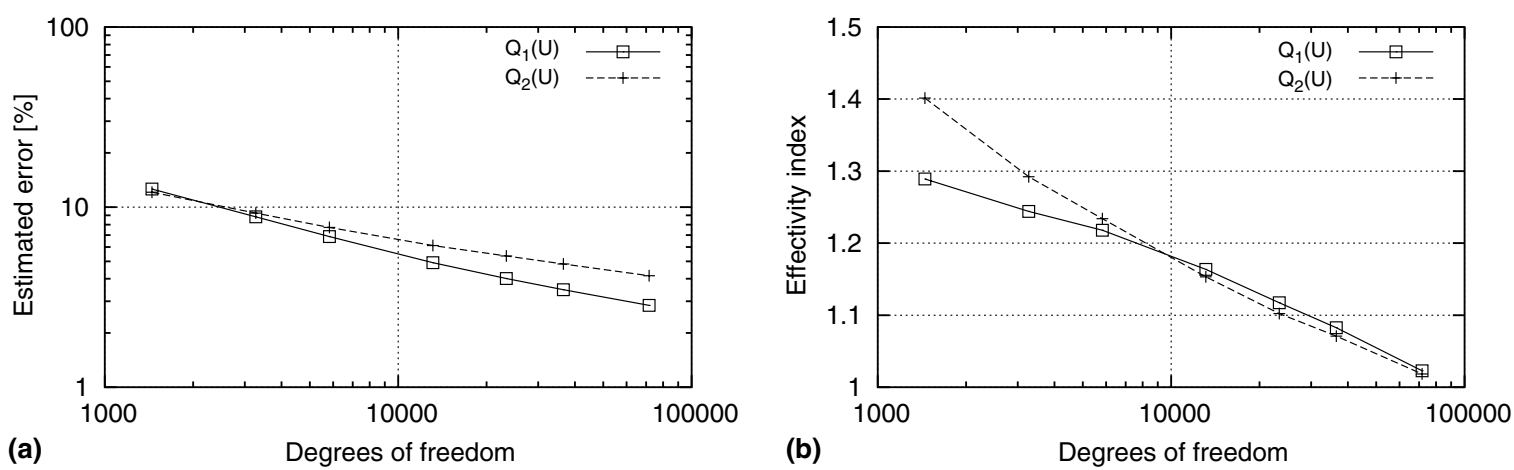

Fig. 3. Results of goal-oriented error estimation for the backward facing step problem (linear Stokes flow and rigid cantilever) for the quantities of interest defined in (65) and (66) using the approximate error representation (53) and employing for each estimate the same fluid mesh of 4-node FCBI elements and 9-node Galerkin elements: (a) estimated (absolute) relative errors in the quantities of interest, (b) corresponding effectivity indices. The numbers of degrees of freedom refer to the uniformly refined 4-node FCBI element meshes with the first mesh as shown in Fig. 1(b) and correspond to the velocity and pressure degrees of freedom.

\subsection{Flow over backward facing step and impinging on a flexible cantilever}

We again consider the model problem in Fig. 1 but now we assume the cantilever to be flexible undergoing large deformations (plane strain state) and the fluid to be governed by the incompressible Navier-Stokes equations. In our analysis we use the following parameters:

Structure: Young's modulus $E=1.0 \mathrm{E} 7$; Poisson's ratio $v=0.3$,

Fluid: Viscosity $\mu=1.0$; Mass density $\rho=100$.

Assuming the step height $(l=2.0)$ to be the characteristic length of the problem yields the Reynolds number $R e=200$.

The quantities of interest are, as defined in (65) and (66), the horizontal tractions across the cantilever located at $y=16.0$ and the resulting bending moment at its fixed end.

For our goal-oriented error estimation we use the scheme proposed in (64). The start-mesh is now shown in Fig. 4, and this mesh is, in succession, uniformly refined. The reference solution was obtained using again the uniform mesh of 31,232 9-node Galerkin finite elements with 280,334 degrees of freedom for the fluid and now a fine mesh of 9-node elements for the cantilever structure. The resulting tip displacement was 0.593 (see also Section 4.3).

In the analyses we employ for the fluid flow the 4-node FCBI element and the 9-node Galerkin element, always using the same meshes. As shown in Fig. 5, the estimated relative percentage error decreases reasonably and the corresponding effectivity indices tend to 1.0. In the figure the numbers of degrees of freedom refer to the coupled system.

\subsection{Influence of the structural mesh size}

While in Section 4.2 we considered a fixed structural mesh, we also want to study the influence of the structural mesh on the accuracy of the quantities of interest. For this purpose we use the fixed fluid mesh corresponding to the problem solution with 21,532 degrees of freedom referred to in Fig. 5, and vary only the structural mesh. The first structural mesh consists of a discretization using $10 \times 1$ elements (one element in the thickness direction) and this mesh is refined to obtain meshes of $20 \times 2,30 \times 3, \ldots, 80 \times 8$ elements. The variation of the structural mesh will, of course, affect the fluid flow due to the coupling of the fluid and the structure, and hence it will influence the values of the quantities of interest considered in this study.

Fig. 6 shows the results computed using the scheme proposed in (62). As shown in the Figure, and as expected, using quite coarse 9-node element structural meshes gives already very good results, in particular compared to the 4-node element

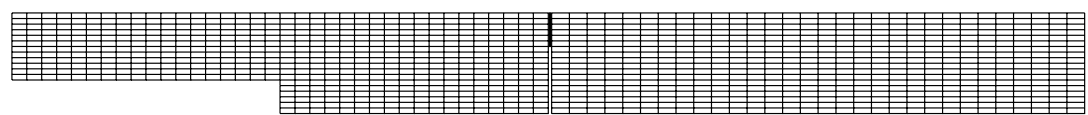

Fig. 4. Start-mesh of four-node FCBI elements used in the backward facing step problem when considering Navier-Stokes fluid flow fully coupled with the flexible cantilever undergoing large deformations, for the quantities of interest defined in (65) and (66). The approximate error representation (64) is used with, for each estimate, the same fluid mesh of 4-node FCBI elements and 9-node Galerkin elements. 

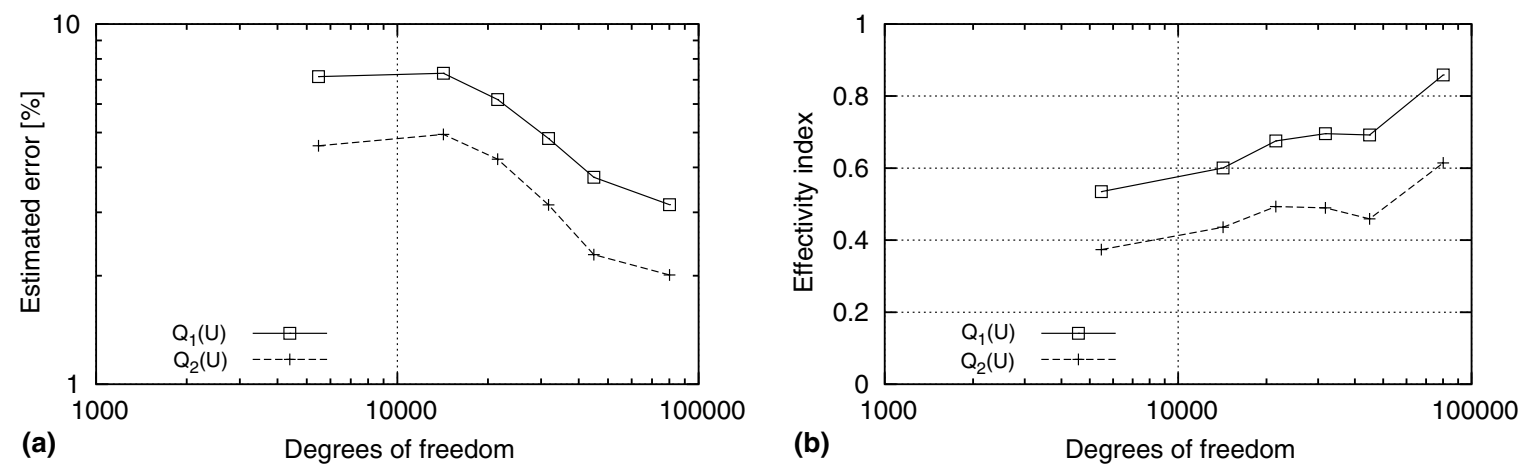

Fig. 5. Results of goal-oriented error estimation for the backward facing step problem when considering Navier-Stokes fluid flow fully coupled with the flexible cantilever undergoing large deformations, for the quantities of interest defined in (65) and (66). The approximate error representation (64) is used with for each estimate the same fluid mesh of 4-node FCBI elements and 9-node Galerkin elements: (a) estimated (absolute) relative errors in the quantities of interest, (b) corresponding effectivity indices. The numbers of degrees of freedom refer to the uniformly refined 4-node FCBI element meshes with the first mesh as shown in Fig. 4 and correspond to the velocity, pressure and displacement degrees of freedom.
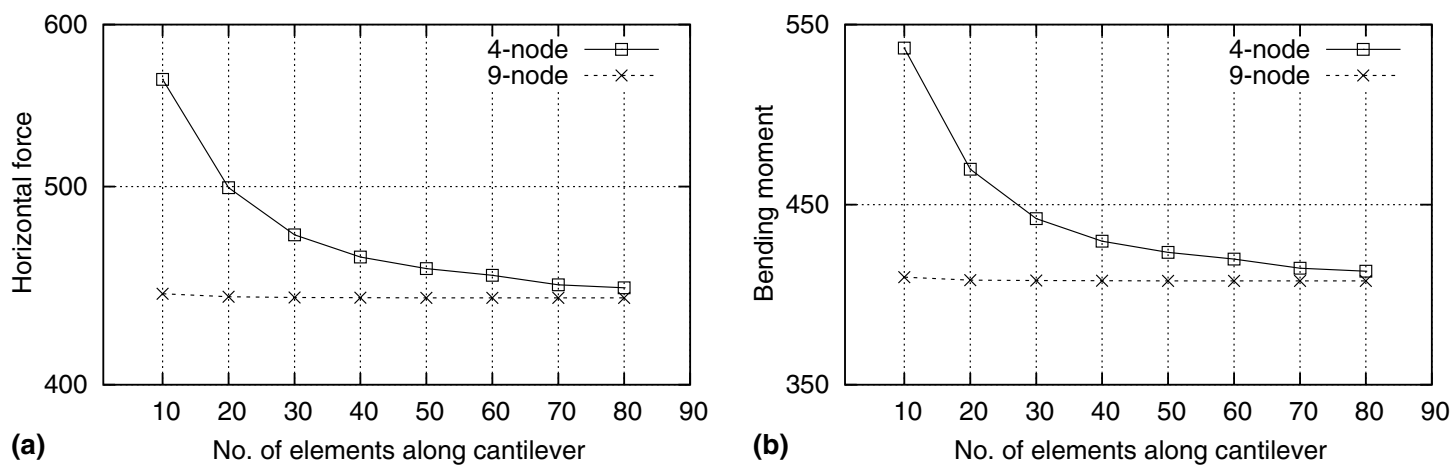

Fig. 6. Results of goal-oriented error estimation for the backward facing step problem when considering Navier-Stokes fluid flow fully coupled with the flexible cantilever undergoing large deformations, for the quantities of interest defined in (65) and (66), with the approximate error representation (64), using for each estimate the same fluid mesh of 4-node FCBI elements (corresponding to the third solution in Fig. 5) and varying the structural mesh: (a) horizontal force, (b) bending moment at the support of the cantilever. The given number of elements for the structural mesh refers to the number of elements used along the cantilever where $1 / 10$ of that number is used for discretization in the thickness direction of the cantilever.

structural mesh solutions. We conclude that the accuracy of the quantity of interest, as considered in this study, mainly depends on the fluid mesh size, while the structural mesh can be quite coarse provided 9-node elements are used.

\section{Conclusions}

We presented some developments for the goal-oriented error estimation in fluid flow structure interaction analyses. In many such interaction analyses the focus is on the structural response and the aim is only to solve accurately for the maximum forces and moments in the structure. The fluid flow needs to be predicted just sufficiently well to reach an accurate response prediction of the structure. Hence the fluid mesh can probably in principle be rather coarse-provided the fluid flow analysis procedures admit such meshes -when compared to a fine fluid mesh used to obtain a detailed and accurate fluid flow response.

The use of goal-oriented error estimation is therefore quite appealing: the goal quantities are the bending moments and shear forces at critical locations in the structure. We developed in this paper some governing equations for the error estimation when general structural models and Navier-Stokes fluids are considered. We also presented a few illustrative solutions. The basic premise is to significantly reduce the computational effort in the fluid flow structure interaction solution, while also have an error control on the quantities of interest. This reduction in solution effort might be particularly important when an optimization of the structure is pursued, which might entail many repeated fluid flow structure interaction solutions.

The field of fluid flow structure interaction analysis is very large, and the developments reported in this paper are only some basic steps considering formulations and implementations for goal-oriented solutions. Clearly, the procedures proposed and used in this paper need to be much more researched, in particular with respect to the effectivity indices, tested, 
evaluated, and refined, possibly using dual problems, considering general flows and large deformation elastic and inelastic response of structures. Also, automatic re-meshing is necessary for the full power of the goal-oriented approach to be harnessed, and the performance of the proposed local error indicators should be investigated. However, we can already conclude that there is considerable potential in the general approach we have considered in this research.

\section{Acknowledgments}

We would like to thank Donald Estep, Colorado State University, Mats Larson, Umea University, and Tinsley Oden, University of Texas at Austin, for their valuable comments on this paper.

The work of Thomas Grätsch was supported by the German Research Foundation (DFG) under contract GR 1894/2-2. We are grateful for this support.

\section{References}

[1] J.H. Argyris, S. Kelsey, Energy theorems and structural analysis, Aircraft Engineering, vols. 26-27 (October 1954 to May 1955), Part I is by J.H. Argyris, and Part II is by J.H. Argyris and S. Kelsey.

[2] J.H. Argyris, Continua and discontinua, in: Proc. of the Conference on Matrix Methods in Structural Mechanics (Wright-Patterson A.F.B., Ohio, 1965) 11-189.

[3] K.J. Bathe (Ed.), Computational Fluid and Solid Mechanics 2003, Elsevier, 2003.

[4] K.J. Bathe (Ed.), Computational Fluid and Solid Mechanics 2005, Elsevier, 2005.

[5] W.B. Shangguan, Z.H. Lu, Experimental study and simulation of a hydraulic engine mount with fully coupled fluid structure interaction finite element analysis model, Comput. Struct. 82 (2004) 1751-1771.

[6] H. Zhang, X. Zhang, S. Ji, Y. Guo, G. Ledezma, N. Elabbasi, H. deCougny, Recent development of fluid-structure interaction capabilities in the ADINA system, Comput. Struct. 81 (2003) 1071-1085.

[7] M.R. Kaazempur-Mofrad, M. Bathe, H. Karcher, H.F. Younis, H.C. Seong, E.B. Shim, R.C. Chan, D.P. Hinton, A.G. Isasi, A. Upadhyaya, M.J. Powers, L.G. Griffith, R.D. Kamm, Role of simulation in understanding biological systems, Comput. Struct. 81 (2003) 715-726.

[8] K.J. Bathe, J. Pontaza, A flow-condition-based interpolation mixed finite element procedure for higher Reynolds number fluid flow, Math. Models Methods Appl. Sci. 12 (2002) 1-15.

[9] K.J. Bathe, H. Zhang, A flow-condition-based interpolation finite element procedure for incompressible fluid flows, Comput. Struct. 80 (2002) 12671277.

[10] H. Kohno, K.J. Bathe, Insight into the flow-condition-based interpolation finite element approach: solution of steady-state advection-diffusion problems, Int. J. Numer. Methods Engrg. 63 (2005) 197-217.

[11] I. Babuška, A.D. Miller, The post-processing approach in the finite element method, I: calculations of displacements, stresses and other higher derivatives of the displacement, Int. J. Numer. Methods Engrg. 20 (1984) 1085-1109.

[12] D. Estep, A posteriori error bounds and global error control for approximations of ordinary differential equations, SIAM J. Numer. Anal. 32 (1995) 148.

[13] K. Eriksson, D. Estep, P. Hansbo, C. Johnson, Introduction to adaptive methods for differential equations, Acta Numer. 4 (1995) 105-158.

[14] R. Becker, R. Rannacher, A feed-back approach to error control in finite element methods: basic analysis and examples, East-West J. Numer. Math. 4 (1996) 237-264.

[15] R. Rannacher, F.T. Suttmeier, A feed-back approach to error control in finite element methods: application to linear elasticity, Comput. Mech. 19 (1997) 434-446.

[16] M. Paraschivoiu, J. Peraire, A. Patera, A posteriori finite element bounds for linear-functional outputs of elliptic partial differential equations, Comput. Methods Appl. Mech. Engrg. 150 (1997) 289-312.

[17] M. Paraschivoiu, A. Patera, A hierarchical duality approach to bounds for the outputs of partial differential equations, Comput. Methods Appl. Mech. Engrg. 158 (1998) 389-407.

[18] J.T. Oden, S. Prudhomme, Goal-oriented error estimation and adaptivity for the finite element method, Comput. Math. Appl. 41 (2001) $735-756$.

[19] W. Bangerth, R. Rannacher, Adaptive Finite Element Methods for Differential Equations, Birkhäuser, Basel, 2003.

[20] T. Grätsch, K.J. Bathe, A posteriori error estimation techniques in practical finite element analysis, Comput. Struct. 83 (2005) $235-265$.

[21] T. Grätsch, K.J. Bathe, Influence functions and goal-oriented error estimation for finite element analysis of shell structures, Int. J. Numer. Methods Engrg. 63 (2005) 631-788.

[22] J.T. Oden, S. Prudhomme, Estimation of modeling error in computational mechanics, J. Comput. Phys. 182 (2002) $496-515$.

[23] K.J. Bathe, ADINA system, Encycloped. Math. 11 (1997) 33-35, see also <www.adina.com>.

[24] K.J. Bathe, H. Zhang, Finite element developments for general fluid flows with structural interactions, Int. J. Numer. Methods Engrg. 60 (2004) $213-232$.

[25] K.J. Bathe, Finite Element Procedures, Prentice Hall, Upper Saddle River, NJ, USA, 1996.

[26] H. Kohno, K.J. Bathe, A 9-node quadrilateral FCBI element for incompressible Navier-Stokes flows, Commun. Numer. Methods Engrg., submitted for publication.

[27] H. Kohno, K.J. Bathe, A flow-condition-based interpolation finite element procedure for triangular grids, Int. J. Numer. Methods Fluids 49 (2005) 849-875.

[28] B. Banijamali, K.J. Bathe, The CIP method embedded in finite element discretizations of incompressible fluid flows, to be submitted. 